\title{
2-S26-1 Symposium26
}

\section{Coordinated regulation of lipid metabolism and inflammatory response in macrophages}

\section{Yumiko Oishi}

Dept. of biochemistry and molecular biology, Nippon Medical School

Growing evidence has suggested that chronic inflammation is important for the pathogenesis of numerous diseases, including metabolic disorders and atherosclerosis. Macrophages play pivotal roles in chronic inflammation. We found that macrophages switch their cellular metabolism and functional phenotype throughout the course of inflammatory response. In response to inflammatory activation via Toll-like receptor (TLR4), macrophages rapidly activate glycolysis, increase inflammatory cytokine expression, acquire M1-like, pro-inflammatory phenotype. By contrast, macrophages increase unsaturated, anti-inflammatory fatty acid synthesis to show M2-like, anti-inflammatory phenotype at 24 hours following TLR4 activation. This late program of anti-inflammatory fatty acid biosynthesis is dependent on SREBP1 and results in the uncoupling of NF-kB binding from gene activation. Consistent with this, anti-inflammatory omega-3 fatty acids are decreased in SREBP $\digamma^{\prime}$ macrophages, and systemic inflammation was prolonged in $S R E B P 1^{-}$mice. These findings suggest the functional switch from M1-like to M2-like, and the metabolic switch from glycolysis to lipid metabolism are tightly linked and coordinately regulated during inflammatory response, and these temporal regulatory programs are important for proper inflammatory activation and resolution. Collectively, macrophages have endogenous, temporal programs to switch their function by linking inflammatory signals, and cellular metabolism. This program would be novel therapeutic target for atherosclerosis and metabolic syndrome. 